\title{
The self-consistent calculation of the edge states at quantum Hall effect (QHE) based Mach-Zehnder interferometers (MZI)
}

\author{
A. Siddiki ${ }^{\mathrm{a}}$, A.E. Kavruk ${ }^{\mathrm{b}, *}$, T. Öztürk ${ }^{\mathrm{b}}, \ddot{U} \cdot \operatorname{Atav}^{\mathrm{b}}$, M. Şahin ${ }^{\mathrm{b}}$, T. Hakioğlu ${ }^{\mathrm{c}}$ \\ ${ }^{a}$ Physics Department, Arnold Sommerfeld Center for Theoretical Physics, and Center for NanoScience Ludwig-Maximilians-Universität München, \\ D-80333 Munich, Germany \\ ${ }^{\mathrm{b}}$ Physics Department, Selcuk University, 42075 Konya, Turkey \\ ${ }^{\mathrm{c}}$ Department of Physics and UNAM Material Science and Nanotechnology Research Institute Bilkent University, 06800 Ankara, Turkey
}

Available online 22 September 2007

\begin{abstract}
The spatial distribution of the incompressible edge states (IES) is obtained for a geometry which is topologically equivalent to an electronic Mach-Zehnder interferometer, taking into account the electron-electron interactions within a Hartree type self-consistent model. The magnetic field dependence of these IES is investigated and it is found that an interference pattern may be observed if two IES merge or come very close, near the quantum point contacts. Our calculations demonstrate that, being in a quantized Hall plateau does not guarantee observing the interference behavior.
\end{abstract}

(C) 2007 Elsevier B.V. All rights reserved.

PACS: 73.20.Dx; 73.40.Hm; 73.50.-h; 73.61.-r

Keywords: Edge states; Quantum Hall effect; Screening; Electronic Mach-Zehnder interferometer

The puzzling interference patterns observed at the quantum Hall effect (QHE) based electronic Mach-Zehnder interferometers (MZI) [1] setups have already attracted many theoreticians to investigate the structure of the "edge states" at these samples. The realistic modelling of the electrostatic potential and electronic density distributions is believed to be indispensable in understanding the rearrangement of the edge states involved. Therefore, the electron-electron interaction has been proposed $[2,3]$ as a possible source of dephasing in these experiments. It was stated that, the conventional edge state explanation of the QHE, i.e. Landauer-Büttiker (LB) formalism, fails to cover the experimental findings. On the other hand, a detailed analysis of the QHE related physics, taking account the formation of the incompressible strips, is needed for a direct comparison with experimental data. Recently, a two channel edge state model is proposed [3], which is able to explain the observed visibility oscillations

\footnotetext{
${ }^{*}$ Corresponding author. Tel.: + 903322231845 .

E-mail address: aekavruk@selcuk.edu.tr (A.E. Kavruk).
}

in terms of a non-Gaussian noise. The essential parameters are the electron velocity and the coupling strength between the "interference" and "detector" channels. In this paper, we extend a previous work [4] to investigate the electrostatics of e-MZI setups in the integer QHE, assuming a topologically equivalent geometry to the experimental one [5]. We aim to provide explicit calculations of the spatial rearrangement of the incompressible edge states [6]. The widely used self-consistent Thomas-Fermi-Poisson screening theory [4] is used to obtain the electron density and electrostatic potential. We propose two possible scenarios to observe interference, depending on the distribution of the incompressible strips, in other words depending on the magnetic field strength.

To obtain the confinement potential, we follow the procedure proposed by Ref. [7]. In this model the bare potential can be obtained at the level of two dimensional electron system (2DES), i.e. in the plane of $z=z_{0}$ measured from the surface into the sample, provided that the surface gate pattern and the potential distribution are known. The contribution of the gates to the total potential at the 2DES 
is given by

$V_{\text {gate }}\left(\mathbf{r}, z_{0}\right)=\frac{1}{\kappa} \int \frac{\left|z_{0}\right|}{2 \pi\left(z_{0}^{2}+\left|\mathbf{r}-\mathbf{r}^{\prime}\right|^{2}\right)^{3 / 2}} V_{\mathrm{g}}\left(\mathbf{r}^{\prime}, 0\right) \mathrm{d} \mathbf{r}^{\prime}$,

where $V_{\mathrm{g}}\left(\mathbf{r}^{\prime}, 0\right)$ is the potential on the sample surface. The second contribution to the external potential comes from the donors, which we simulate by a half-period cosine function in the $y$ direction. Given the external potential in the plane of $2 \mathrm{DES} ; V_{\text {ext }}\left(\mathbf{r}, z_{0}\right)=V_{\text {gate }}\left(\mathbf{r}, z_{0}\right)+V_{\text {donor }}\left(\mathbf{r}, z_{0}\right)$, $\left(x, y, z_{0}\right)=\left(\mathbf{r}, z_{0}\right)$, in the real space, it is straightforward to calculate the screened potential (again in the real space) at $T=0$ and $B=0$, making two dimensional forward and back Fourier transform, using $V_{\text {scr }}(q)=V_{\text {ext }}(q) / \varepsilon(q)$, where $\varepsilon(q)$ is the momentum $(q)$ dependent Thomas-Fermi dielectric function defined by $\varepsilon(q)=1+2 / a_{\mathrm{B}}^{*}|q|$, and $a_{\mathrm{B}}^{*}$ is the effective Bohr radius $(\sim 10 \mathrm{~nm})$. We use this screened potential as an initial input for the following set of two selfconsistent (SC) equations:

$n_{\mathrm{el}}(\mathbf{r})=\int \mathrm{d} E D(E) f\left(\left[E+V(\mathbf{r})-\mu^{\star}\right] / k_{\mathrm{B}} T\right)$,

for the spinless electron density, where $D(E)$ is the bare Landau density of states (DOS), $f(\alpha)=\left[1+e^{\alpha}\right]^{-1}$ the Fermi function and the Hartree potential energy of an electron

$V_{H}(\mathbf{r})=\frac{2 e^{2}}{\bar{\kappa}} \int_{A} \mathrm{~d} \mathbf{r}^{\prime} K\left(\mathbf{r}, \mathbf{r}^{\prime}\right) n_{\mathrm{el}}\left(\mathbf{r}^{\prime}\right)$,

within the Thomas-Fermi approximation. In the case of periodic boundary conditions, as we consider, the kernel $K\left(\mathbf{r}, \mathbf{r}^{\prime}\right)$ can be expressed in an analytically closed form [8], otherwise has to be obtained numerically. The total potential energy is obviously nothing but the sum of Hartree and external potential energies. In the conventional edge state explanation of the integer QHE, one counts the number of the LB ES, which essentially gives the plateau number with an integer filling factor. Without assuming any sort of localization or disorder, the Hall

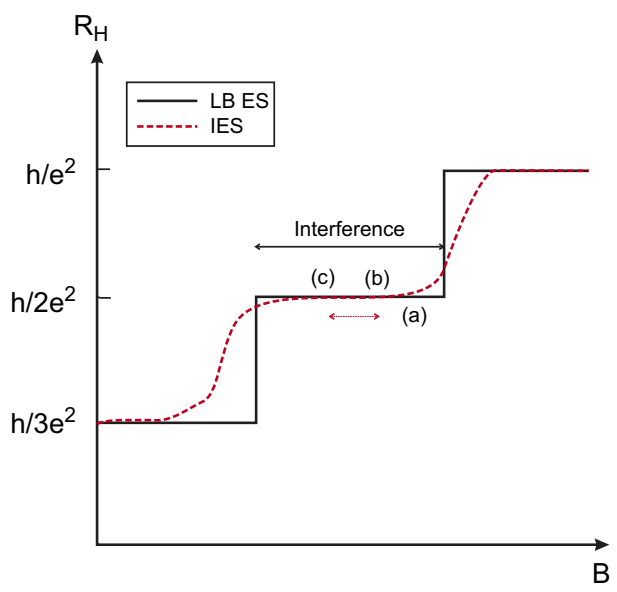

Fig. 1. The sketch of the Hall resistance, considering LB ES (solid line) and incompressible ES (broken line). Expected interference intervals of the magnetic field are denoted by the lines with arrows on both ends. resistance looks like a staircase (cf. Fig. 1), whereas longitudinal resistance exhibits delta spikes at transitions as a function of the $B$ field. This implies that, whenever one enters to a plateau region one always have a percolating LB ES from source to drain, therefore the interference pattern should be observed throughout all the plateau region, contradicting with the experimental findings. Although such a discrepancy can be removed by a large amount of (asymmetric) Landau level broadening, the high mobility of the sample rules out this possibility. On the other hand, in the screening theory of the QHE [6,9] a plateau occurs only if at least an IES exists along the current direction. Within this localization free model, the widths of the plateaus are limited by the thicknesses of the IES, depending on the $B$ field and/or the long-range part of the disorder [9]. In Fig. 2, we plot the electron distribution as a function of the spatial coordinates, calculated within the above SC scheme for a typical Fermi energy, $E_{\mathrm{F}}=$ $12.75 \mathrm{meV}$ at $1 \mathrm{~K}$. The color gradient depicts from zero (dark) to high electron concentration. The high potential bias at the surface guarantees that no electrons can reside under the gates, whereas the light (yellow) stripes highlight
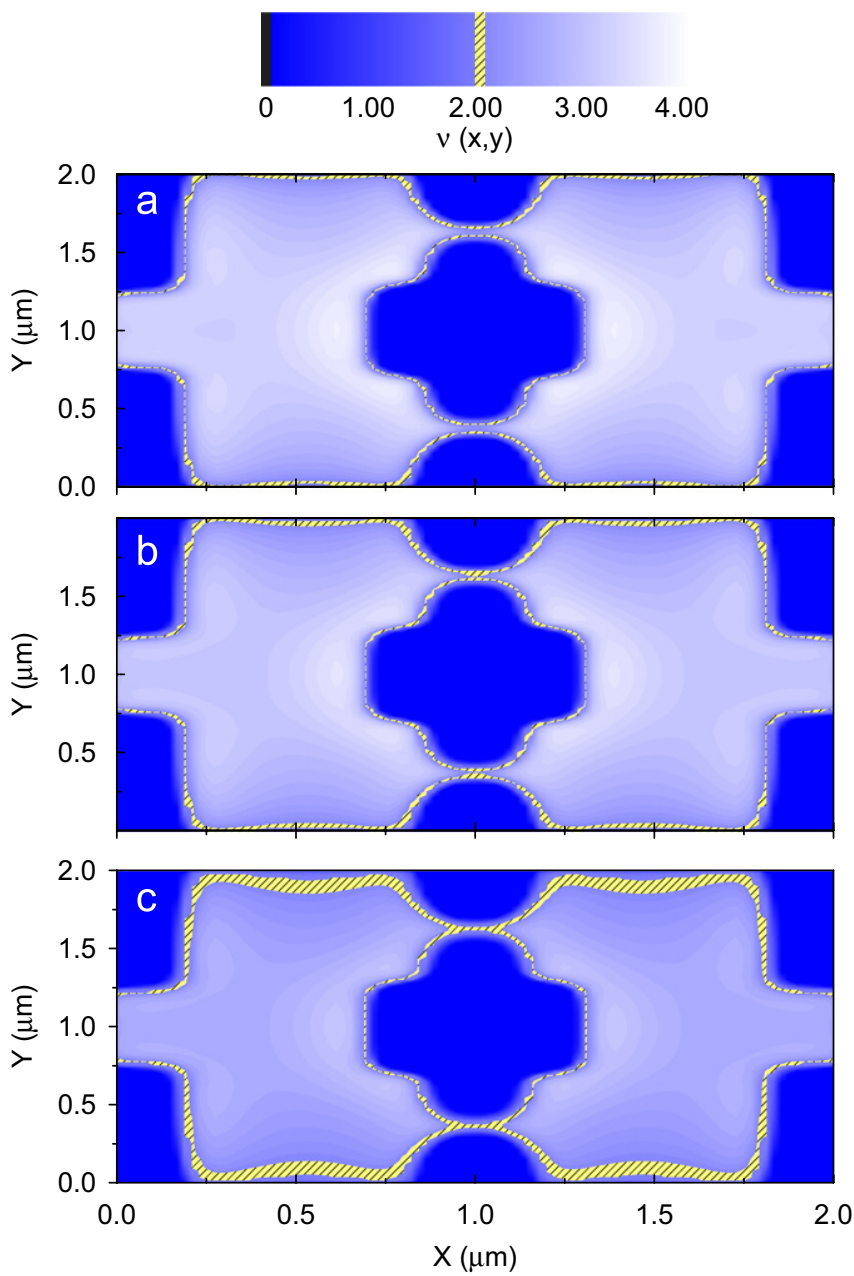

Fig. 2. The color coded $v(x, y)$ for $\Omega=1.15,0.95,0.90$. The calculations are done at $\Omega / k_{\mathrm{B}} T \approx 0.025$. The gates defining the geometry, are taken to be $85 \mathrm{~nm}$ above the 2 DES and biased with $-1.0 \mathrm{~V}$. 
the positions of local filling factor two. Under the conditions considered at the top most panel of Fig. 2 the system will be observed to be almost entering to the plateau region since there exists two (almost) percolating IES (at the top and the bottom part of the geometry), however, we believe that the visibility will be either too small to observe (due to scattering at the constriction or at the bulk) or will be zero. The middle panel shows a situation that, the percolation of the IES is well formed and the system is on the plateau, on contrast the visibility will still be small, since the interference will continue to be dominated by the tunnelling where scattering processes may take place. For the lowest $B$ field, the IES merge at the quantum constrictions and decoherence is minimized, therefore the visibility is predicted to be the highest at a reasonably high mobility sample, similar to the samples measured at the experiments [1,3]. We believe that, if (not only if) the nondissipative current is confined to the IES, where no backscattering takes place, the observed amplitude variation of the visibility as a function of $B$ field at the experiments can simply be explained by an emerging IES at the QPCs. This claim also promotes the fact that, in such sensitive experiments the geometrical shape of the QPCs may play an important role $[4,10]$, although the transmission amplitude remains unchanged.

In summary, the spatial distribution of the backscattering free IES at a MZI (topologically equivalent geometry) is studied, exploiting the smooth variation of the external potential, within the Thomas-Fermi approximation. We have shown that, one would not observe visibility oscillations on each and single magnetic value of the quantized Hall plateau interval in contrast to the LB ES model. We have reasoned this on the base of merging IES at the QPCs and highlighting the importance of the geometry of the constriction. The amplitude itself and the interference interval clearly depend on the mobility of the sample, therefore (also including spin) an extension of this present work may help in improving the sample design and the quality of the observed quantities.

The authors acknowledge the support of the Marmaris Institute of Theoretical and Applied Physics (ITAP), TUBITAK Grant 105T110, and Selcuk University BAP contracts $07101003 \& 07101037$, SFB631 and DIP.

\section{References}

[1] Y. Ji, et al., Nature 422 (2003) 415; I. Neder, et al., Phys. Rev. Lett. 96 (2006) 016804

[2] F. Marquardt, Europhys. Lett. 72 (2005) 788.

[3] I. Neder, F. Marquardt, New J. Phys. 9 (2007) 112.

[4] A. Siddiki, F. Marquardt, Phys. Rev. B 75 (2007) 045325.

[5] P. Samuelsson, E. Sukhorukov, M. Buttiker, Phys. Rev. Lett. 92 (2004) 026805.

[6] A. Siddiki, R.R. Gerhardts, Phys. Rev. B 70 (2004) 195335.

[7] J.H. Davies, I.A. Larkin, Phys. Rev. B 49 (1994) 4800.

[8] P.M. Morse, H. Feshbach, Methods of Theoretical Physics, McGraw-Hill, New York, 1953 Band II, p. 1240.

[9] A. Siddiki, R.R. Gerhardts, Int. J. Mod. Phys. B 21 (2007) 1362.

[10] A. Siddiki, et al., Physica E (2007), doi:10.1016/j.physe.2007.08.097. 